\title{
Rotational properties of dipolar Bose-Einstein condensates confined in anisotropic harmonic potentials
}

\author{
F. Malet ${ }^{1}$, T. Kristensen ${ }^{2}$, S. M. Reimann ${ }^{1}$, and G. M. Kavoulakis ${ }^{3}$ \\ ${ }^{1}$ Mathematical Physics, Lund Institute of Technology, P.O. Box 118, SE-22100 Lund, Sweden \\ ${ }^{2}$ École Normale Supérieure de Cachan, Cachan cedex, 94230 Cachan, France \\ ${ }^{3}$ Technological Educational Institute of Crete, P.O. Box 1939, GR-71004, Heraklion, Greece
}

(Dated: June 26, 2018)

\begin{abstract}
We study the rotational properties of a dipolar Bose-Einstein condensate confined in a quasi-twodimensional anisotropic trap, for an arbitrary orientation of the dipoles with respect to their plane of motion. Within the mean-field approximation we find that the lowest-energy state of the system depends strongly on the relative strength between the dipolar and the contact interactions, as well as on the size and the orientation of the dipoles, and the size and the orientation of the deformation of the trapping potential.

PACS numbers: 67.85.De 05.30.Jp 67.85.Jk
\end{abstract}

\section{INTRODUCTION}

The rotational properties of Bose-Einstein condensates have been studied extensively over the last decades, beginning with the well-known experiments performed on liquid Helium. Bose-Einstein-condensed gases of ultracold atoms have provided us with an ideal system for the study of this problem [1-3]: In addition to the fact that they are dilute, many of the system parameters are tunable externally, making it possible to explore experimentally many novel properties. So far most of the experiments have been performed in isotropic [4 11], as well as anisotropic 12 harmonic potentials. More recently, other trapping potentials such as, for example, anharmonic [13] and toroidal [14] ones, have also been addressed.

While the initial experiments considered atoms with interactions that could be modelled via an isotropic and local, i.e., contact, potential, more recently the behavior of atoms with an electric or magnetic dipole moment has been investigated [15, 16]. The physics of these gases is rather different because the dipolar interaction is anisotropic, non-local, and finally it can be both attractive - when the dipoles are placed head-to-tail - and repulsive - when they are placed side-to-side. Initially dipolar effects were investigated experimentally in ${ }^{52} \mathrm{Cr}$ atoms [17]. More recently dipolar effects have also been investigated experimentally in condensates of ${ }^{39} \mathrm{~K}$ and ${ }^{7} \mathrm{Li}$ 18 atoms, in spinor condensates 19], in ultracold gases of Dy atoms [20], and in ultracold polar molecules [21, 22]. These studies have focused on different aspects of the dipolar interaction such as, e.g., its anisotropy [18], or on its attractive character and the consequent possible collapse of the gas 23].

The rotational properties of Bose-Einstein condensates are described e.g. in the review articles [1 $[3]$, as well as in Refs. 24 30]. In particular, anisotropic trapping potentials have been considered in Refs. 26 30]. Dipolar gases have also been extensively addressed theoretically, see for example Refs. [15, 16, 31 39], and studies on dipolar Bose-Einstein condensates under rotation have shown that the vortex properties of these systems are strongly affected by the anisotropic character of the dipole-dipole interaction and by the relative strength between the dipolar and the contact interactions [34, 36, 37, 40, 41]. The relative orientation of the dipoles with respect to the confining potential also affects the critical rotation frequency for the nucleation of a vortex state in the condensate, lowering (raising) its value with respect to the non-dipolar case when the trap is elongated in the direction perpendicular (parallel) to the orientation of the dipoles [35].

Some of the above studies have employed a fully threedimensional approach to solve the Gross-Pitaevskii equation [35, 37], whereas other works have examined quasione- or quasi-two-dimensional systems [34, 36, 38, 39]. In most of these papers, the orientation of the dipoles has been limited to only two possibilities: perpendicular, or parallel to their plane of motion. Very recently, the more general and interesting case of an arbitrary orientation of the dipole moment of the atoms has been investigated for non-rotating gases [38, 39, 42].

Here, we study the rotational properties of a quasi-twodimensional dipolar Bose-Einstein condensate confined in a rotating anisotropic harmonic trapping potential, with an arbitrary orientation of the dipoles. Since the latter can be controlled by means of external electric or magnetic fields, this is also very relevant from the experimental point of view. As compared to the case where the gas is trapped in an axially-symmetric trap and interacts with a contact potential, here the symmetry is broken in two separate and independent ways: Through the anisotropy of the trap, and also through the directional dependence of the dipolar interaction. The combination of these two symmetry-breaking mechanisms gives rise to interesting vortex configurations, which are investigated in this article.

In what follows we first present our model in Sec. II. In Sec. III we investigate the rotational properties of the system as a function of the trap anisotropy, the strength of the dipolar interaction, and the orientation of the dipole moment of the atoms with respect to their plane of motion. Finally, in Sec. IV we summarize our results and 


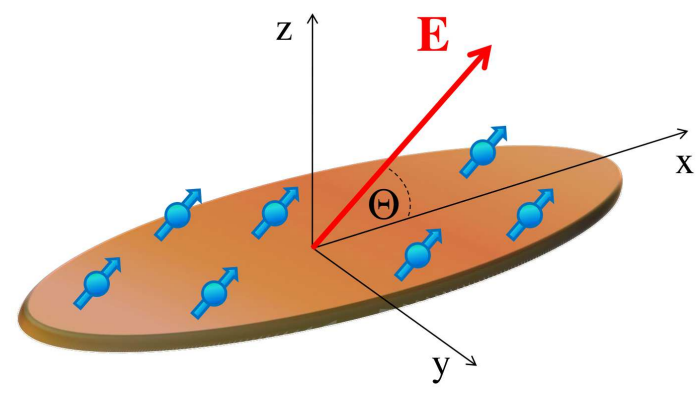

FIG. 1: (Color online) Schematic illustration of the quasitwo-dimensional elliptical confining potential. A very tight potential is assumed along the $z$ axis (not shown in the plot). The atoms have a dipole moment in the $x z$ plane, at some direction that forms an angle $\Theta$ with the $x$ axis, due to the action of an external electric (or magnetic) field $\mathbf{E}$.

conclude.

\section{MODEL AND METHODOLOGY}

Let us consider a Bose-Einstein condensate of atoms of mass $M$ confined by some external potential, which is harmonic in all directions,

$$
V_{T}(\mathbf{r})=\frac{1}{2} M \omega_{z}^{2} z^{2}+V\left(\mathbf{r}_{\perp}\right) .
$$

Here $\omega_{z}$ is the frequency of the potential along the $z$ axis, which we assume to be very tight, while in the $x y$ plane the potential is taken to be anisotropic,

$$
\begin{aligned}
V\left(\mathbf{r}_{\perp}\right) & =\frac{1}{2} M \omega_{0}^{2}\left(x^{2}+y^{2}\right)+2 A M \omega_{0}^{2}\left(x^{2}-y^{2}\right) \\
& \equiv \frac{1}{2} M\left(\omega_{x}^{2} x^{2}+\omega_{y}^{2} y^{2}\right) .
\end{aligned}
$$

In the above expression $\mathbf{r}_{\perp}=(x, y)$, and $\omega_{x} \equiv$ $\omega_{0} \sqrt{1+4 A}, \omega_{y} \equiv \omega_{0} \sqrt{1-4 A}$ are the trap frequencies along the $x$ and $y$ axes, respectively. Here $A$ is a dimensionless parameter determining both the direction and the strength of the deformation of the trap.

The assumption of a very tight potential along the $z$ axis, $\hbar \omega_{z}$ being much larger than any other energy scale in the problem, allows us to treat the problem as quasitwo-dimensional. With this assumption, the degrees of freedom along the $z$ axis are frozen and the system occupies only the lowest-energy eigenstate $\phi_{0}(z)$ of the potential $V(z)=M \omega_{z}^{2} z^{2} / 2$. As a result, the order parameter $\Psi(\mathbf{r})$ separates to $\Psi(\mathbf{r})=\psi\left(\mathbf{r}_{\perp}\right) \phi_{0}(z)$. We also assume that the atoms have a non-zero (electric or magnetic) dipole moment, and that all the dipoles are aligned to some external (electric or magnetic) field, which is in the $x z$ plane, and forms an angle $\Theta$ with the $x$ axis, as illustrated schematically in Fig.1. In three dimensions, the dipolar interaction between two atoms separated by the vector $\mathbf{r}$ is given by 16

$$
V_{d d}(\mathbf{r})=D^{2} \frac{1-3 \cos ^{2} \theta_{r d}}{r^{3}} .
$$

In the above expression, $D^{2}=d^{2} /\left(4 \pi \epsilon_{0}\right)$ when the atoms have an electric dipole moment $d$, where $\epsilon_{0}$ is the permittivity of the vacuum. When the atoms have a magnetic moment $\mu, D^{2}=\mu_{0} \mu^{2} /(4 \pi)$, where $\mu_{0}$ is the permeability of the vacuum. Also, $\theta_{r d}$ is the angle between the polarization direction and the relative position vector $\mathbf{r}$ of the two dipoles.

As shown in Refs. [38, 39], in order to derive an effective potential for the two-dimensional problem, one assumes the Gaussian profile of the harmonic oscillator in the $z$ direction $\phi_{0}(z)$. Then, integrating $V_{d d}(\mathbf{r})$ over the $z$ coordinates,

$$
V_{\text {eff }}\left(\mathbf{r}_{\perp}\right)=\int\left|\phi_{0}(z)\right|^{2} V_{d d}\left(\mathbf{r}-\mathbf{r}^{\prime}\right)\left|\phi_{0}\left(z^{\prime}\right)\right|^{2} d z d z^{\prime} .
$$

Working with cylindrical polar coordinates $\mathbf{r}_{\perp}=\left(r_{\perp}, \phi\right)$,

$$
\begin{array}{r}
V_{\text {eff }}\left(\mathbf{r}_{\perp}\right)=\frac{D^{2}}{\sqrt{8 \pi}} \frac{e^{w / 2}}{a_{z}^{3}}\left\{(2+2 w) K_{0}(w / 2)-2 w K_{1}(w / 2)\right. \\
+\cos ^{2} \Theta\left[-(3+2 w) K_{0}(w / 2)+(1+2 w) K_{1}(w / 2)\right] \\
\left.+2 \cos ^{2} \Theta \cos ^{2} \phi\left[-w K_{0}(w / 2)+(w-1) K_{1}(w / 2)\right]\right\} .(5)
\end{array}
$$

Here $a_{z}=\sqrt{\hbar / m \omega_{z}}$ is the oscillator length in the $z$ direction, $w \equiv r_{\perp}^{2} / 2 a_{z}^{2}$, and $K_{0}(w)$ and $K_{1}(w)$ are the zero-order and first-order modified Bessel functions of the second kind. It can be checked easily that for the particular cases $\Theta=0^{\circ}$ and $90^{\circ}$, Eq. (5) reduces to the expressions found in Refs. [34, 36]. The assumed quasitwo-dimensional behavior of the system allows us to derive the above expression for the dipolar interaction, and also allows us to consider any arbitrary orientation of the dipoles.

In addition to the dipolar interaction, we also consider the usual contact potential between the atoms, given by $V_{\text {int }}(\mathbf{r})=U_{0} \delta(\mathbf{r})$, where $U_{0}=4 \pi \hbar^{2} a / M$. Here $a$ is the scattering length for zero-energy elastic atom-atom collisions. Again, integrating over the profile along the $z$ axis, the corresponding effective two-dimensional contact potential becomes $V_{s}\left(\mathbf{r}_{\perp}\right)=g \delta\left(\mathbf{r}_{\perp}\right)$, where $g$ is given by $g=U_{0} \int\left|\phi_{0}(z)\right|^{4} d z=U_{0} /\left(\sqrt{2 \pi} a_{z}\right)$.

Assuming also that the trap rotates around the $z$ axis with some angular frequency $\Omega$, the Gross-Pitaevskii equation for the order parameter $\psi\left(\mathbf{r}_{\perp}\right)$ takes the following, nonlocal form

$$
\begin{aligned}
{\left[-\frac{\hbar^{2} \nabla_{\perp}^{2}}{2 M}+V\left(\mathbf{r}_{\perp}\right)+V_{\mathrm{dip}}\left(\mathbf{r}_{\perp}\right)+g\left|\psi\left(\mathbf{r}_{\perp}\right)\right|^{2}\right.} & \left.-\Omega L_{z}\right] \psi\left(\mathbf{r}_{\perp}\right) \\
& =\mu \psi\left(\mathbf{r}_{\perp}\right),(6)
\end{aligned}
$$

where $L_{z}$ is the operator of the angular momentum along the $z$ axis, $\mu$ is the chemical potential, and

$$
V_{\mathrm{dip}}\left(\mathbf{r}_{\perp}\right)=\int V_{\mathrm{eff}}\left(\mathbf{r}_{\perp}-\mathbf{r}_{\perp}^{\prime}\right)\left|\psi\left(\mathbf{r}_{\perp}^{\prime}\right)\right|^{2} d \mathbf{r}_{\perp}^{\prime}
$$


is the dipolar interaction potential. We have treated this term making use of the convolution theorem and fastFourier-transform techniques, combined with the introduction of a cutoff at small distances, where $V_{\text {eff }}\left(\mathbf{r}_{\perp}\right)$ diverges, as done in Ref. [32].

In order to solve Eq. (6) we have employed a fourthorder split-step Fourier method within an imaginary-time propagation approach [43]. Thus, starting with a reasonable initial state we propagate it in imaginary time until we reach a steady state, after a sufficiently large number of time steps.

\section{RESULTS - ROTATIONAL PROPERTIES OF A DRIVEN GAS}

Since there is a large number of parameters in the present problem, we fix $\omega_{z}$ and $\omega_{0}$ throughout this study, choosing $\omega_{z} / \omega_{0}=100$.

One useful dimensionless quantity is the ratio between the interaction energy per particle due to the contact potential $E_{s}$ and the oscillator energy $\hbar \omega_{0}$. For a cloud of homogeneous density of radius equal to the oscillator length $a_{0}=\sqrt{\hbar / M \omega_{0}}$ the interaction energy is $E_{s}=g N /\left(\pi a_{0}^{2}\right)$, where $N$ is the atom number. The ratio $E_{s} / \hbar \omega_{0}$ is thus equal to $g N M /\left(\pi \hbar^{2}\right)$, and in what follows we choose it equal to $100 / \pi$.

Another dimensionless quantity that is convenient to introduce is the ratio between the "dipolar length" $a_{d d}$, and the $s$-wave scattering length $a$, which we denote as $\varepsilon_{d d} \equiv a_{d d} / a[16]$. Defining $a_{d d} \equiv M D^{2} /\left(3 \hbar^{2}\right)$, then $\varepsilon_{d d}=$ $4 \pi D^{2} / 3 U_{0}$. Since $U_{0}=\sqrt{2 \pi} g a_{z}, \varepsilon_{d d}$ is also equal to $(\sqrt{8 \pi} / 3) D^{2} /\left(g a_{z}\right)$. This quantity gives roughly the ratio between the expectation value of the dipolar energy $E_{\text {dip }}$ and $E_{s}$. Therefore, with the above choice of parameters, and for the specific values of $D$ that we choose below, we have the following hierarchy of energy scales:

$$
\hbar \omega_{z}>E_{s} \gtrsim E_{\text {dip }}>\hbar \omega_{0}
$$

It should be noted that the stability of the system is already a non-trivial question [44, 45], as the dipole-dipole interaction is partly attractive and one has to be cautious, since the system may be unstable against collapse. We have observed this collapse in our calculations, although not in the range of parameters corresponding to the figures shown below.

\section{A. Vortex configurations as a function of the dipole strength and the dipole orientation}

Let us first study the rotational properties of the system as a function of the orientation of the dipoles of the atoms with respect to their plane of motion, and of the dipolar strength. In Fig. 2 we show the two-dimensional atom density for $\Theta=0,15,30,45$, and 90 degrees, and $\varepsilon_{d d}=0,0.31,0.38,0.54$, and 0.67 . We also choose a

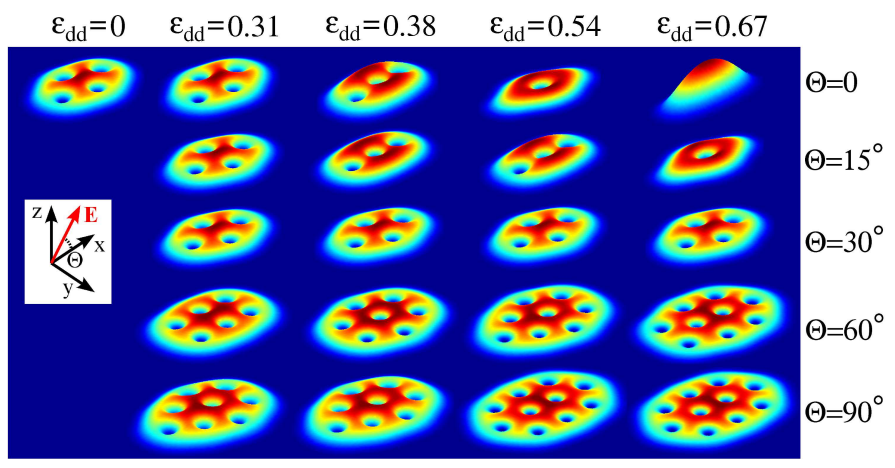

FIG. 2: (Color online) Two-dimensional atom density as a function of the strength of the dipolar interaction, for $\varepsilon_{d d}=$ $0,0.31,0.38,0.54$, and 0.67 , and of the orientation of the dipoles, for $\Theta=0,15,30,60$, and 90 degrees, with the nondipolar case $\left(\varepsilon_{d d}=0\right)$ also shown as a reference. The trap deformation is $A=-0.03$, and the angular velocity of the trap is $\Omega / \omega_{0}=3 / 4$. The inset illustrates the coordinate axes and the external field.

moderate value of $A=-0.03$, which corresponds to a trapping potential that is more tight along the $y$ axis and finally $\Omega / \omega_{0}$ is set equal to $3 / 4$. From Fig. 2 one can see that the way the vortex structure changes as a function of $\varepsilon_{d d}$ depends sensitively on the orientation of the dipoles. For example, when the polarization is along the $x$ axis $\left(\Theta=0^{\circ}\right)$ the attractive part of the dipolar interaction gives rise to a well-known self-induced squeezing of the density in the $y$ direction [37] that reduces progressively the number of vortices as $\varepsilon_{d d}$ increases.

Thus, the four vortices present in the non-dipolar case $\left(\varepsilon_{d d}=0\right)$ and for $\varepsilon_{d d}=0.31$, get reduced to three and to one for $\varepsilon_{d d}=0.38$ and 0.54 , respectively, with a structure that clearly reflects the symmetry imposed by the external field along the $x$ axis. Finally, when the dipolar strength exceeds a certain value $\left(\varepsilon_{d d} \simeq 0.67\right)$, the width of the gas becomes too small to accommodate any vortex state. A similar effect has been found in nondipolar condensates which are confined in anisotropic potentials, where there is a critical deformation of the trap beyond which all the vortices are expelled from the cloud [26, 29, 30].

When the polarization angle becomes $\Theta=15^{\circ}$ we find a similar, but less pronounced disappearance of the vortex lattice, as in this case the attractive part of the dipolar interaction becomes less important. For $\Theta=30^{\circ}$, however, the repulsive and the attractive parts of the dipole interaction become comparable, and as a result the vortex structure is not affected by the increase in $\varepsilon_{d d}$ within the range that we have considered.

When the angle exceeds $60^{\circ}$ the behavior of the system changes. In this case, the repulsive part of the interaction becomes dominant, giving rise to a stronger net repulsion. As a consequence, the number of vortices increases with increasing $\varepsilon_{d d}$. This effect is maximal when the dipoles are polarized along the $z$ axis $\left(\Theta=90^{\circ}\right)$, in which case the dipolar interaction is purely repulsive and isotropic. 


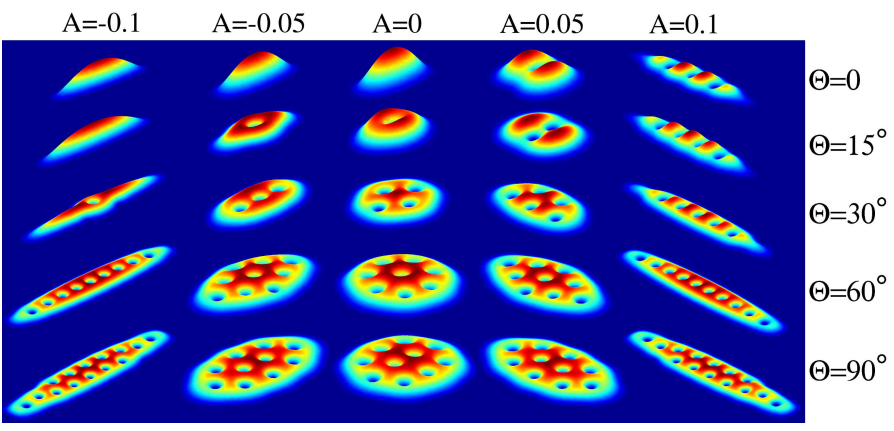

FIG. 3: (Color online) Two-dimensional atom density as a function of the ellipticity of the trap for $A=-0.1,-0.05,0$, 0.05 and 0.1 , and of the orientation of the dipoles, for $\Theta=0$, 15, 30, 60 and 90 degrees. The angular velocity of the trap is $\Omega / \omega_{0}=3 / 4$, and $\varepsilon_{d d}$ is fixed to 0.67 . The coordinate axes correspond to the same as in Fig. 2.

B. Vortex configurations as a function of the ellipticity of the trap and the dipole orientation

We have also studied the rotational behavior of the system as a function of the trap deformation $A$ and of the angle $\Theta$, for a fixed dipolar strength $\varepsilon_{d d}=0.67$ and a fixed rotational frequency of the trap, $\Omega / \omega_{0}=3 / 4$, as shown in Fig. 3. In particular, we considered the cases of strong and moderate deformation of the trap in the $y$ axis $(A=-0.1$ and $A=-0.05$, respectively), the non-deformed case $(A=0)$, and of strong and moderate deformation along the $x$ direction $(A=0.1$ and $A=0.05$, respectively). When the dipoles are polarized in-plane, along the $x$ axis $\left(\Theta=0^{\circ}\right)$, the squeezing of the density in the $y$ direction reported in the previous subsection due to the attractive part of the dipolar interaction prevents the formation of vortices, not only for $A<0$, but also in the non-deformed case $(A=0)$. However, when the trap is squeezed in the direction of the polarization of the dipoles, the confinement competes with the dipolar interaction, forcing the cloud to expand along the $y$ axis, and thus allowing the formation of vortices in the system, with their number increasing as the trap gets more deformed.

The same qualitative behavior is found when the polarization angle $\Theta$ is slightly increased. Indeed, for $\Theta=15^{\circ}$ the main difference with the previous case is that the cloud has a vortex state for $A=0$ and $A=-0.05$. Similarly, when the angle becomes 30 degrees, the tendency of reducing (increasing) the number of vortices when the trap is squeezed in the $y(x)$ axis is still clear, although in this case the vortex lattice does not disappear completely, even for the largest deformation that we have considered.

This trend is however no longer observed for $\Theta=60^{\circ}$. In this case, the dipolar interaction is mostly repulsive and the number of vortices increases with respect to the non-deformed case, regardless of the sign of the deformation. When the latter is moderate, the vortex structure is the same for $A= \pm 0.05$, and differences are only present in the case of strong deformation. Finally, when the dipoles are polarized along the vertical direction, i.e., when $\Theta=90^{\circ}$, the dipolar interaction is isotropic and purely repulsive and as a consequence the densities are axially symmetric for $A=0$ and have a mirror symmetry with respect the interchange of the $x$ and $y$ axes in the deformed cases.

\section{Angular momentum versus the rotational frequency of the trap}

Finally, we have studied the expectation value of the angular momentum of the system in the $z$ direction, $\left\langle L_{z}\right\rangle$, as a function of the rotation frequency $\Omega$, for different values of $\Theta$ and $A$, and for a fixed strength of the dipolar interaction $\varepsilon_{d d}=0.67$, as shown in Fig. 4 .

The different cases shown in Fig. 4 share some common characteristics. First of all, they are consistent with the divergence of the angular momentum when $\Omega \rightarrow \min \left\{\omega_{x}, \omega_{y}\right\}$, i.e. when $\Omega \rightarrow \omega_{y}=\omega_{0} \sqrt{1-4 A}$ since $A$ is chosen to be positive. Also, as $\Omega / \omega_{0}$ approaches the limiting value $\sqrt{1-4 A}$, the curves become more smooth, as the number of vortices increases and the system becomes more classical, resembling in this limit solid-body rotation. We also observe in Fig. 4 that for some given $\Omega$ and $A$, as $\Theta$ decreases, the angular momentum of the gas decreases as well.

In addition, the results in Fig. 4 are consistent with the intuitive expectation that the more the system is distorted from axial symmetry (i.e., as $A$ increases, or as $\Theta$ decreases), the more it resists in accommodating a vortex state [24], and as a result the critical frequency $\Omega_{c}$ necessary for vortex nucleation increases. This effect is most clearly seen in Fig. 5 , which shows the critical value of $\Omega$ for the nucleation of the first vortex state as a function of $\Theta$. This increase of $\Omega_{c}$ is associated with a distortion of the axial symmetry of the cloud, which may carry angular momentum even when it is vortex-free, as required by the irrotational nature of the velocity field.

In most cases shown in Fig. 4 there is a discontinuous increase of $\left\langle L_{z}\right\rangle$ with increasing $\Omega$, which is associated with the progressive entry of vortex states into the gas, very much as in the case of non-dipolar atoms confined in an isotropic trapping potential 25]. One interesting exception is shown in the top panel of Fig. 4, where for $\Theta=0^{\circ}$ (i.e., when the dipolar interaction breaks the axial symmetry in the most extreme way) the angular momentum increases continuously from zero. In this range of $\Omega / \omega_{0}$ the density of the cloud is elongated along the direction of the polarizing field, breaking the axial symmetry of the potential, and it is also vortex-free. Beyond a critical rotational frequency $\left(\Omega / \omega_{0} \approx 0.82\right)$, however, the angular momentum shows a sudden increase, which is associated with three vortices entering the cloud. On the contrary, for the other values of $A=0.03$ and 0.1 that we have considered, there is a discontinuous transition to a state with one vortex, as seen in the central and 


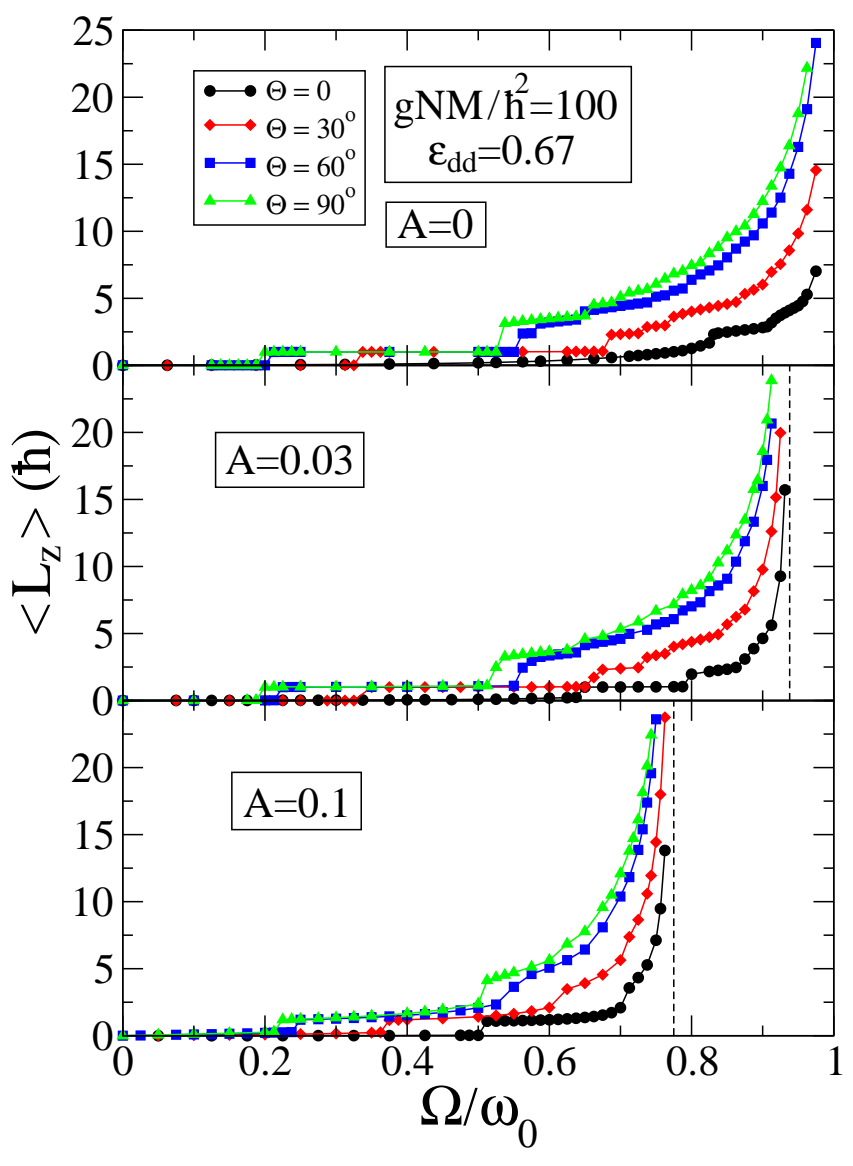

FIG. 4: (Color online) Expectation value of the angular momentum of the gas as a function of the rotational frequency of the trap, for $A=0$ (top), $A=0.03$ (middle) and $A=0.1$ (bottom), for $\varepsilon_{d d}=0.67$, and $\Theta=0,30,60$, and 90 degrees. The dashed vertical lines in the lower two curves correspond to the value $\Omega / \omega_{0}=\sqrt{1-4 A}$.

bottom panels of Fig. 4 , where $\left\langle L_{z}\right\rangle$ jumps from zero to unity.

\section{SUMMARY AND CONCLUSIONS}

In this paper we have investigated the stationary states of a dipolar Bose-Einstein condensate that is confined in a rotating, elliptical, quasi-two-dimensional trapping potential. In particular, we examined this problem as a function of the orientation of the dipole moment of the atoms, of the relative strength between the dipolar and the contact interactions, and of the deformation of the trap.

While a repulsive contact potential between the atoms tends to spread the gas uniformly, the trap deformation and the anisotropic character of the dipolar interaction favor the breaking of the axial symmetry. These two mechanisms give rise to interesting configurations, which we have investigated here. For example, at a fixed trap deformation, we observe either the disappearance of the

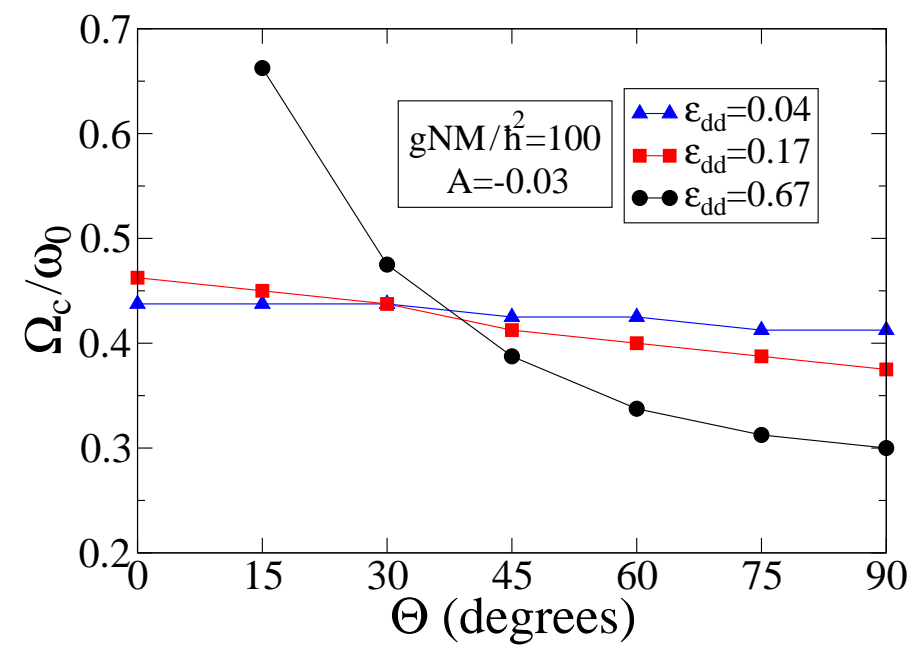

FIG. 5: (Color online) Critical rotational frequency of the trap for the nucleation of the first vortex as a function of $\Theta$, for $A=-0.03$, and for $\varepsilon_{d d}=0.04,0.17$, and 0.67 .

vortex lattice, or nucleation of vortices as the dipolar strength increases, depending on the orientation of the dipoles. A similar behavior is found if we fix the strength of the dipole interaction and we vary the orientation of the dipole moments, or the deformation of the trap.

Another interesting observation is that even in the case of an axially-symmetric trapping potential, relatively weak values of the dipolar interaction energy (as compared to the energy due to the contact potential), suffice to give rise to rather significant effects due to the breaking of the axial symmetry. An analogous result has been found in the case of dipole-free atoms that rotate in an asymmetric trapping potential, where even weak deviations from axial symmetry have a substantial effect [26].

One rather general and intuitive observation that results from the present study is that the more the gas is distorted from axial symmetry (via either the deformation of the trap, or via the tilt of the angle of the dipoles from the direction perpendicularly to their plane of motion), the more it expels the vortices, with the critical frequency for vortex nucleation being increased [24].

The strength of the dipolar interaction affects the critical frequency for the formation of vortices in the cloud in a non-monotonic way. When the dipoles are oriented along their plane of motion, or close to this direction, the rule mentioned in the previous paragraph applies and, and as a result, the critical rotational frequency for vortex nucleation increases with the dipolar interaction strength. On the other hand, when the dipoles are either perpendicular to their plane of motion, or close to that direction, the critical rotational frequency for vortex nucleation decreases as the dipolar interaction increases. As noticed in Ref. [35] this is due to the fact that when, for example, $\Theta=90^{\circ}$, the dipolar interaction is purely repulsive and isotropic, and as a result the average atom den- 
sity becomes lower. While the dipolar interaction affects the large-scale properties of the cloud (i.e., its radius), it also leaves its small-scale properties (i.e., the coherence length) unaffected, which is reflected in the decrease of $\Omega_{c}$.

Our study gives just a flavor of the richness of the physical effects that result mainly from the anisotropic nature of the dipolar interaction, but also from the deformation of the trapping potential. In particular, the sensitivity of the behavior of the gas on the orientation of the external polarizing field (and thus of the dipole moment of the atoms), which may be tuned easily, is remarkable.
The experimental investigation of these effects appears therefore worthwhile.

\section{ACKNOWLEDGEMENTS}

We thank Niels Søndegaard for valuable assistance in the FFT treatment of the dipolar interaction. We also thank Manuel Barranco, Georg Bruun, Jonas Cremon, and Martí Pi for useful discussions. This work was financed by the Swedish Research Council.
[1] A. J. Leggett, Rev. Mod. Phys. 73, 307 (2001).

[2] Alexander Fetter, Rev. Mod. Phys. 81, 647 (2009).

[3] H. Saarikoski, S. M. Reimann, A. Harju, and M. Manninen, Rev. Mod. Phys. 82, 2785 (2010).

[4] M. R. Matthews, B. P. Anderson, P. C. Haljan, D. S. Hall, C. E. Wieman, and E. A. Cornell, Phys. Rev. Lett. 83, 2498 (1999).

[5] K. W. Madison, F. Chevy, W. Wohlleben, and J. Dalibard, Phys. Rev. Lett. 84, 806 (2000).

[6] F. Chevy, K. W. Madison, and J. Dalibard, Phys. Rev. Lett. 85, 2223 (2000).

[7] K. W. Madison, F. Chevy, V. Bretin, and J. Dalibard, Phys. Rev. Lett. 86, 4443 (2001).

[8] J. R. Abo-Shaeer, C. Raman, J. M. Vogels, and W. Ketterle, Science 292, 476 (2001).

[9] P. C. Haljan, I. Coddington, P. Engels, and E. A. Cornell, Phys. Rev. Lett. 87, 210403 (2001).

[10] P. Engels, I. Coddington, P. C. Haljan, and E. A. Cornell, Phys. Rev. Lett. 89, 100403 (2002).

[11] P. Engels, I. Coddington, P. C. Haljan, V. Schweikhard, and E. A. Cornell, Phys. Rev. Lett. 90, 170405 (2003).

[12] E. Hodby, G. Hechenblaikner, S. A. Hopkins, O. M. Maragò, and C. J. Foot, Phys. Rev. Lett. 88, 010405 (2001).

[13] V. Bretin, S. Stock, Y. Seurin, and J. Dalibard, Phys. Rev. Lett. 92, 050403 (2004).

[14] C. Ryu, M. F. Andersen, P. Cladé, Vasant Natarajan, K. Helmerson, and W. D. Phillips, Phys. Rev. Lett. 99, 260401 (2007).

[15] M. A. Baranov, Phys. Rep. 464, 71 (2008).

[16] T. Lahaye, C. Menotti, L. Santos, M. Lewenstein, and T. Pfau, Rep. Prog. Phys. 72, 126401 (2009).

[17] Axel Griesmaier, Jörg Werner, Sven Hensler, Jürgen Stuhler, and Tilman Pfau, Phys. Rev. Lett. 94, 160401 (2005).

[18] M. Fattori, G. Roati, B. Deissler, C. D'Errico, M. Zaccanti, M. Jona-Lasinio, L. Santos, M. Inguscio, and G. Modugno, Phys. Rev. Lett. 101, 190405 (2008); S. E. Pollack, D. Dries, M. Junker, Y. P. Chen, T. A. Corcovilos, and R. G. Hulet, Phys. Rev. Lett. 102, 090402 (2009).

[19] M. Vengalattore, S. R. Leslie, J. Guzman, and D. M. Stamper-Kurn, Phys. Rev. Lett. 100, 170403 (2008).

[20] M. Lu, S. H. Youn, and B. L. Lev, Phys. Rev. Lett. 104, 063001 (2010).

[21] C. Ospelkaus, S. Ospelkaus, L. Humbert, P. Ernst, K.
Sengstock, and K. Bongs, Phys. Rev. Lett. 97, 120402 (2006).

[22] S. Ospelkaus, A. Pe'er, K.-K. Ni, J. J. Zirbel, B. Neyenhuis, S. Kotochigova, P. S. Julienne, J. Ye, and D. S. Kin, Nature Phys. 4, 622 (2008).

[23] T. Koch, T. Lahaye, J. Metz, B. Fröhlich, A. Griesmaier, and T. Pfau, Nature Phys. 4, 218 (2008).

[24] Alexander L. Fetter, J. of Low Temp. Phys. 16, 533 (1974).

[25] D. A. Butts, and D. S. Rokhsar, Nature (London) 397, 327 (1999).

[26] Marion Linn, Matthias Niemeyer, and Alexander L. Fetter, Phys. Rev. A 64, 023602 (2001).

[27] M. Ö. Oktel, Phys. Rev. A 69, 023618 (2004).

[28] S. Sinha and G. V. Shlyapnikov, Phys. Rev. Lett. 94, 150401 (2005).

[29] Amandine Aftalion, Xavier Blanc, and Nicolas Lerner, Phys. Rev. A 79, 011603(R) (2009).

[30] Alexander L. Fetter, Phys. Rev. A 81, 033620 (2010).

[31] L. Santos, G. V. Shlyapnikov, P. Zoller, and M. Lewenstein, Phys. Rev. Lett. 85, 1791 (2000).

[32] K. Góral, K. Rzazewski and T. Pfau, Phys. Rev. A 61, 051601(R) (2000); K. Góral and L. Santos, Phys. Rev. A 66, 023613 (2002).

[33] S. Giovanazzi, A. Görlitz, and T. Pfau, Phys. Rev. Lett. 89, 130401 (2002).

[34] S. Yi and H. Pu, Phys. Rev. A 73, 061602(R) (2006).

[35] D. H. J. O'Dell and C. Eberlein, Phys. Rev. A 75, 013604 (2007).

[36] S. Komineas and N. R. Cooper, Phys. Rev. A 75, 023623 (2007).

[37] M. Abad, M. Guilleumas, R. Mayol, M. Pi, and D. M. Jezek, Phys. Rev. A 79, 063622 (2009).

[38] Yongyong Cai, Matthias Rosenkranz, Zhen Lei, and Weizhu Bao, Phys. Rev. A 82, 043623 (2010).

[39] J. C. Cremon, G. M. Bruun, and S. M. Reimann, Phys. Rev. Lett. 105, 255301 (2010).

[40] R. M. W. van Bijnen, D. H. J. O'Dell, N. G. Parker, and A. M. Martin, Phys. Rev. Lett. 98, 150401 (2007); R. M. W. van Bijnen, A. J. Dow, D. H. J. O'Dell, N. G. Parker, and A. M. Martin, Phys. Rev. A 80, 033617 (2009);

[41] Tapio P. Simula, Jukka A. M. Huhtamki, Masahiro Takahashi, Takeshi Mizushima and Kazushige Machida, J. Phys. Soc. Jpn. 80 (2011) 013001.

[42] I. Sapina, T. Dahm, and N. Schopohl, Phys. Rev. A 82, 053620 (2010). 
[43] S. A. Chin and E. Krotscheck, Phys. Rev. E 72, 036705 (2005).

[44] Uwe R. Fischer, Phys. Rev. A 73, 031602(R) (2006).
[45] Ryan M. Wilson, Shai Ronen, and John L. Bohn Phys. Rev. A 80, 023614 (2009). 